\title{
SALUD PUBLICA
}

Rev. Chil, Pediatr, 62 (1); 5660,1991

\section{Percepción matema de la calidad en la atención a niños hospitalizados}

\author{
Freya Fernảndez K. ${ }^{1}$; Rubén Puentes R. ${ }^{1}$; Silvia Quiroga D. ${ }^{1}$; \\ Isabel Salas $A^{1} ;$ Humberto Soriano $B .^{1}$
}

\section{Maternal perception of care's quality to their children during hospitalization}

\begin{abstract}
In order to evaluate the perception of mothers on the care given to their hospitalized chirdren, a survey was specially designed and carried out with 100 mothers on the day of their chi id's discharge. Hospital care was rated excelent or good by a $96 \%$, regular or bad by $4 \%$ of surveyed moihers. Most (74\%) morhers saw a: least one member of the caring team acting affectionately with their children; only $2 \%$ complained that her child was reprimanded or punished. Lack of equipment or human resources and comfort facilit es were frequently percived by mathers but quality of care was generally considered to be satisfactory. This kind of studies is probably usefu! to improve community's health services.
\end{abstract}

(K. $\psi$ words: Delivery of health care, hospitalization, child hospitalized.)

Se sabe que la hospitalización es un riesgo psicológico potencjal ya que priva al niño de las instancias más importantes para su desarrollo, como la familia, la escuela y sus pares ${ }^{1-3}$.

La hospitalización reviste para el niño características amenazantes, ya que debe adaptarse a un medio físico y humano desconocido que incluye experiencias que pueden ser dolorosas y traumáticas $4,5,7-9$.

Cuando los niños, especialmente los más pequeños, vuelven a sus hogares, casi invarjablemente presentan problemas conductuales, cuyos efectos pueden prolongarse hasta la edad adulta 0 adolescencia ${ }^{5-10}$.

La intensidad de estos trastornos varía según la edad del paciente y su madurez cognitiva ${ }^{11,12}$, la enfermedad que motivó su internación, las características de su familia y muy especialmente el trato recibido en el hospital ${ }^{8}$.

En afros recientes ha habido una marcada tendencia a "humanizar" el cuidado de los niños en los hospítales ${ }^{3}$. La encuesta, cuyos resultados se describen a continuación, se realizó con el propósito de conocer cómo percibian las madres la atención recibida por sus hijos en nuestro hospital, con el fin de reunir datos que, junto a la información teórica $\mathrm{y}$ a la obtenida en otras experiencias nacionales ${ }^{7,9}$, contribuyan al diseño

1. Servicio de Pedjatría. Hospital Dr. Sótero del Río. de programas de apoyo a los niños que se encuentren en estas circunstancias.

\section{Material y Método}

Se realizaron 100 encuestas, en forma consecutiva, las que se administraron a las madres al momento de egresar sus hijos del hospital, en los meses de julio y agosto de 1988.

La encuesta fue anónina y se aplicó en forma individual, autoadministrada. Las madres que requirieron ayuda fueron asistidas por personal ajeno al hospital (estudiantes en práctica de psicología), pues el de la institucjón no tuvo conocimiento de su realización.

Las encuestas constaban de 17 preguntas que abarcaban la calidad de la comunicación del médjco, la auxiliar y la enfermera con los padres; la calidad global del trato que recibía el niño; aspectos relacionados con el horario de visitas y percepción respecto a insuficiencias en recursos materiales.

La muestra tomada representó aproximadamente a $5 \%$ del total de egresos anuales del Servicio de Pediatría; $55.1 \%$ de las madres pertenecían al nivel socioeconómico A y $44,8 \%$ al nivel $B$. de la calificación empleada regularmente en establecimientos hospitalarios del Ministerio de Salud de Chile, ambos con ingresos inferiores a $\$ 16.000$ (diez y seis mil pesos, $\mathrm{m} / \mathrm{n}$ de 1990).

\section{Resultados}

La información obtenida sobre la calidad de la comunicación de los padres con el médico, la enfermera y la auxiliar de enfermería, mostró que, al ingreso, $92 \%$ de las madres tuvo 
comunicación con el médico, y que la informaciôn que éste dio fue considerada satisfactoria (figura 1). Esta conversación también se efectuó al alta en $71 \%$ de los casos encuestados. En la descripción de la comunicación las madres po. dían contestar varias opciones. predominando al egreso también las favorables: tranquila $25,6 \%$, clara $31,4 \%$, amable $39,7 \%$.

En $61 \%$ de los casos las madres conversaron con la enfermera durante la hospitalización. Cuando dicha entrevista ocurrió, las madres también la percibieron positivamente (figura 2).

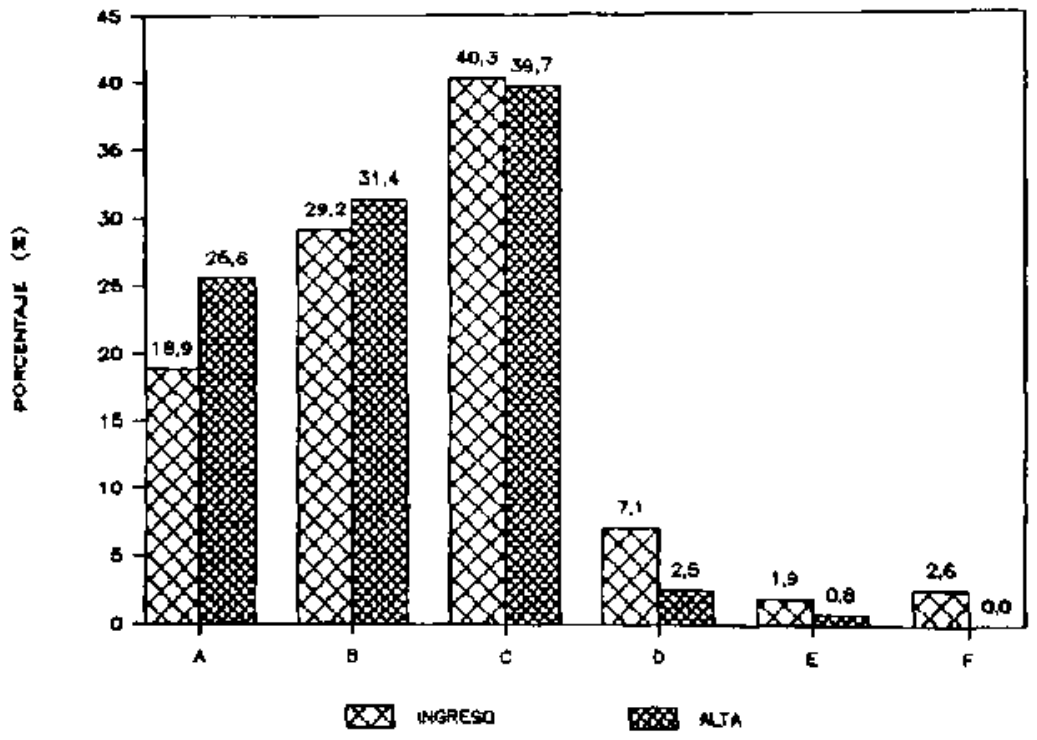

Figura 1: Calidad de la información dada por el médico. A: tranquila. B: clara. C: amable. D: A la rápida. Fi: enredada. F: fría.

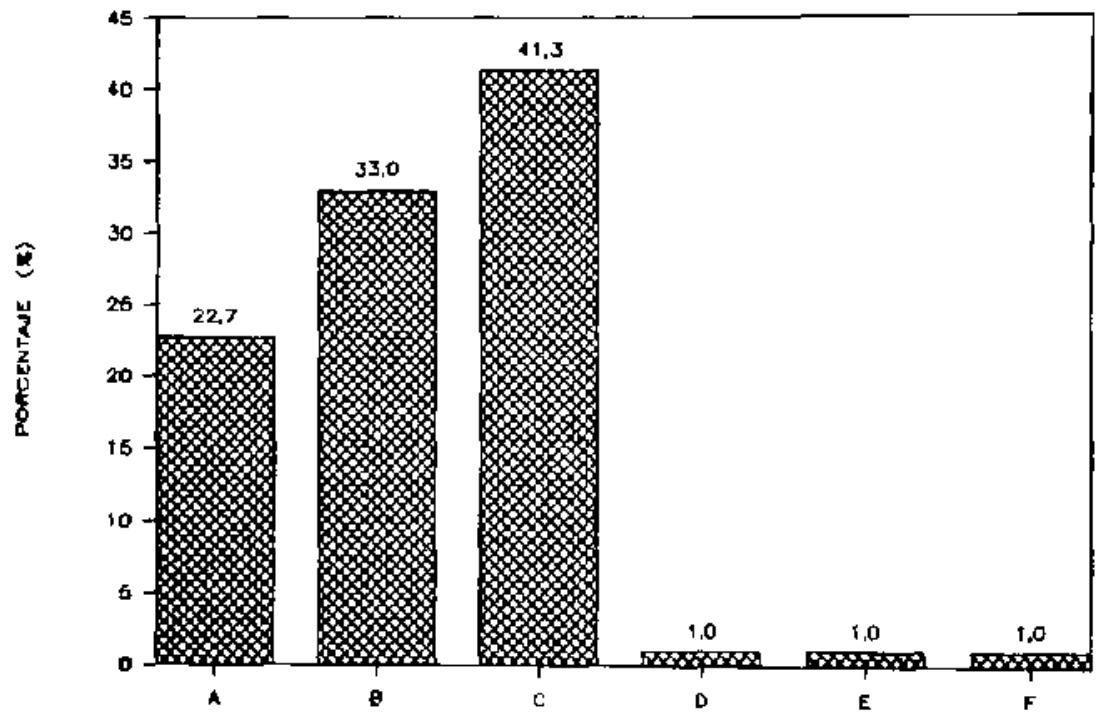

Figura 2: Calidad de la información dada pot la enfermera. A: tranquila. B: clara. C: amable. D: fría. E: rápida. F: enredada. 
La atención de la auxiliar fue muy bien calificada (figura 3); en esta materia las madres podian anotar, además, comentarios. Entre los aspectos favorables más frecuentes se señalaron: trato amable; cariñoso, preocupación por el niño, estaba limpio, se veia ordenado, alimentos al dja, lo encontraban contento, mudado, respondían a las dudas, preguntas y ofrecían explicaciones.

Los comentarios desfavorables fueron más infrecuentes: lo encontraba llorando, sucio, una mamá dijo que la habian insultado, trato frio, no siempre estaban, había demasiada presión asistencial.

Respecto a la calidad del trato que recibió el niño en general las madres lo percibieron como cariñoso $(90 \%$ ). En $50,9 \%$ de los casos, la auxiliar de la sala fue responsable del trato favorable al paciente. Sólo $2 \%$ de las madres refirieron que sus hijos habían sido retados o castigados.

En la figura 4 se resume globalnente cómo percibieron las madres la atención recibida por el niño, destacando que en $96 \%$ de los casos ésta fue catalogada como excelente o buena. En este contenido se ofreció también la posibilidad de respuestas abiertas. Los comentarios favorables mas frecuentes fueron: tratamiinto amable, tenian paciencia, eran cariñosos, se preocupaban por atenderlo, se mejoró, respondian a las preguntas, informaban bien, diaria. mente. Los comentarios desfavorables fueron menos frecuentes: trato frio, descuido, falta de calor humano, pérdida de objetos, informes telefónicos cortos y poco claros, no tranquiliza- ban, poco horario de visita y dificultades para el ingreso de ambos padres.

En cuanto a otros aspectos de la hospitalización, $31 \%$ de las madres refirieron que sus nifros tuvieron problemas con la alimentación, pensando que esto se debió principalmente a la enfermedad y a que no les gustó el tipo de comida.

Tambièn se les consultó cómo habian encontrado a sus hijos cuando los visitaron: $30,7 \%$ los encontró sir hacer nada; $17,1 \%$ jugando con alguien del personal, y $15 \%$ entretenidos con juguetes.

Con respecto al entorno fisico, las madres percibieron falta de adornos en 33,3\% de los casos, de juguetes en $30 \%$ y de ropa en $23,3 \%$, todos relevantes en un hospital para nir̂os. También se refirieron con frecuencia a insuficiencia de personal auxiliar.

El horatio de visita del hospital es de 12 a 16 horas. Generalmente entra uno de los padres. Existen excepciones en los niños desnutridos, los que alimentan a pecho y los pacientes oncológicos a los que se les dan amplias facilidades para ser visitados. En este aspecto, $71 \%$ de las madres encontró adecuado el horario de visita, $24 \%$ escaso y $5 \%$ excesivo.

Estas cifras que hemos analizado correspon. den a los resultados generales obtenidos en todo el servicio de pediatría. Se tabularon también de modo separado según las diferentes unidades: lactantes, infecciosos $y$ segunda infancia, exis. tiendo algunas diferencias dadas por las distintas edades $y$ afecciones de los pacientes. Los

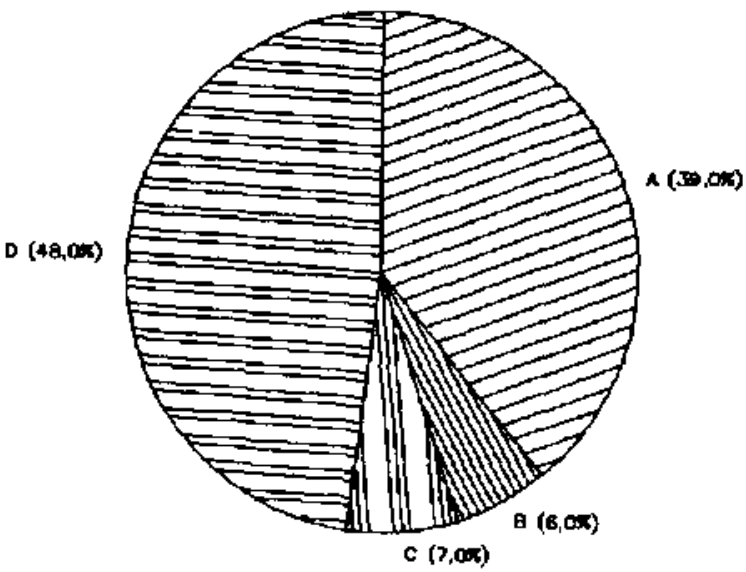

Figura 3.: Percepción de la madre respecto al trato recibido por su hijo de parte de la auxiliar. A: excelente. B: mala. C: regular. D: buena.

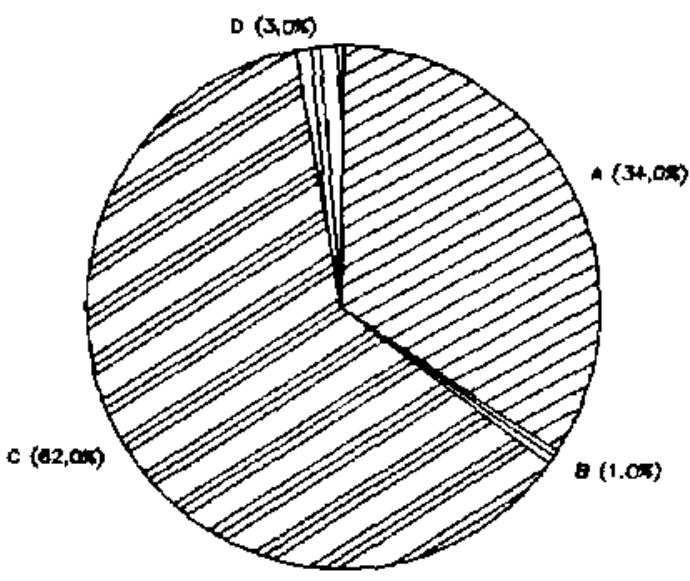

Figura 4: Percepción global de la madre respecto a le atención recibida durante la hospitalización. A: excelente. B: mala. C. Buena, D: regular. 
resultados generales y parciales fueron expuestos y analizados en cada unidad.

\section{Discusión}

Los resultados de la encuesta cumplieron los objetivos para la cual fuera diseñada, permitien. do conocer cómo percibían las madres la aten. ción recibida por sus hijos.

La atención brindada a los niños por este servicio de pediatria fue, en general, estimada como satisfactoria, siendo estos resultados mejores que lo esperado. Esto coincide con otros estudios similares al nuestro ${ }^{13-17}$.

Se consideró especialmente satisfactoria la comunicación con el personal hospitalario, cuando ésta se realizó, destacando la acción que desempeña la auxiliar de enfermería en la aten. ción đel niño hospitalizado. Corno ella esta gran rúmero de horas junto al niño, la calidad de su trato influyó en forma importante en la buena percepción materna del mismo hacia su hịjo.

Aunque los resultados indicaron que la atención brindada a los niños fue considerada satisfactoria, se detectaron varias áreas en las cuales parece importante introducir cambios.

Algunos de ellos tienen solución a corto plazo, como mejorar la comunicación del médico y la enfermera con los pađres del niño y hacer más adecuado el entorno físico. Otras situaciones son más dificiles de cambiar, como la alta presión asistencial y la faita de personal auxiliar.

Es posible que en esta experiencia existan varios factores que podrian haber distorsionado ta percepción de las madres, por ejemplo, bajo nivel de exigencias, desconocimiento de otras realidades con las cuales comparar, ausencia de niños fallecidos en la muestra, dificultad de la madre para expresar sentimientos negativos, etapa de la hospitalización en que fue tomada la encuesta $y$ elementos de juicio insuficientes para valorar la adecuación de algunos contenjdos como exámenes y medicamentos. Por otra parte, la encuesta se realizó en meses de gran presión asistencial (julio, agosto), donde la calidad de la atención podria haberse percibido como más deficiente.

Es importante, además, analizar los resultados a la luz de la información teórica. Por ejemplo, aunque las madres consideraron satisfactorios los horarios de visita, otros estudios indican que los niffos, sobre todo los menores de cuatro años, se benefician permaneciendo el mayor tiempo posible con sus madres ${ }^{3,18}$, por lo que tambiérl puede plantearse como un objetivo alargar el tiempo de visita.

Tomando en cuenta lo expuesto, los resultados de esta encuesta han sido muy valiosos, para contribuir al diseño de un programa de apoyo a] niño hospitalizado, el cual servirá para hacer la estadía de los nirios en nuestro hospital lo menos traumática posible.

Estos resultados han permitido también valorizar y gratificar la labor personal, especialmente la atención de la auxiliar de enfermería, ya que muchas veces ella no percibe lo destacado de su rol, lo que, unido a otros factores, puede incidir en insatisfacción laboral. La información recogida significó para todos un gran estímulo respecto a cómo contribuir a mejorar la interacción con los niños y sus padres y la calidad de atención que se brinde a ellos.

\section{Resumen}

A fin de evaluar la percepción que tienen las madres sobre la calidad de atención a los nifoos hospitalizados se realizó una encuesta especialmente diseñada. Se aplicó en 100 madres, en forma aleatoria, al egreso de los niños. La atención fue encontrada excelente o buena en $96 \%$, regular o mala en $4 \% ; 74 \%$ de las madres vio a alguien del personal, en su mayoría auxiliares de enfermería, actuar cariũosamente con sus hijos. Sólo $2 \%$ afirmó que su hijo fue alguna vez retado o castigado. Al mismo tiempo, se detectaron djversos déficit materiales, de recursos humanos y de la atención y el trato. Se concluye que la atenciôn fue percibida de modo satisfactorio. Se estima necesario continuar este tipo de estudios para perfeccionar la atención en salud.

(Palabras clave: niño hospitalizado, calidad del trato.)

\section{Referencias}

1. Kenny Thomas, J.: The hospitalized child. Pediatr Clin North Am 1975; 22: 583-593.

2. Perry Jones, M.; MacLean, William E.: Children with chronic Illness. Pediat? Clin North Am 1988; 35: 1325-1337. 
3. Robertson, $J$.: Young children in hospizal. $2^{a}$ Edición. Londres; Tavistodk, 1970.

4. Cataldo, M.; Maldonado, $J_{\text {.: }}$ Psychological ef fects of pediatric intensive care on staff, patjents and family. Clapter 41 - Pediatric Intensive Care 1988. Baltimore Williams and Wilkins, editores. $1461-1480$.

5. Adams, $M$ : A hospital play program helping children with serious illness. Am J Orthopsychiat $1976 ; 46: 416-424$.

6. Elander, $G . ;$ Nilsson, A.F.; Lindberg, $T$ : Behaviour in four year-olds who have experienced hospitalization and day care. Am J Orthopsychiat 1986; $56: 612-616$.

7. Anónimo: Informe Programa del Niño Hospitalizado. Manejo del niño hospitalizado. Hospital Luis Calvo Mackenna.

8. Servicio de Neuropsiquiotría Infantil. Programa de apoyo psicológico y pedagógico al niños hospitalizado. Revista Médicł del Hospital Paula Jaracuemada, 1987; $1: 8-15$.

9. Quinton, D.; Rulfer, M.: Early hospital admissions and later disturbances of behaviour. Develop Med Child Neurol 1976; 18 : 447-459.

10. Dougles, J.W.B.: Early hospital admissions and fater disturbances of behaviour and learning.
Deyelopment Med Child Neurot. 1975; 17: 456480.

11. Brewster, A.: Chronically jll hospitalized children's concept of their illness. Pedatrics 1982;69: 355362.

12. Eisen, Ch.: Communicating with sick and hospitaLized chitdren. J Child Psychol Psychiat 1984; 25: $181-189$.

13. Chormey, $E$.: Patient doctor communication. Pediatr Clin North Am 1972:22: 583-593.

14. Korsch, B.: Gozzi. E, Viela, F.: Gasp in doctorpatjent communication. I Doctor patient interaction and patient satisfaction. Pediatrics 1968; $42: 855-870$.

15. Deisher, R.; Willam. L.; Spicholz, R.: Stanjorst, $S$.: Mothers opinion of their pediatric care. Pediatries $1965 ; 35: 82-90$.

16. Marten. George $W_{. ;}$Manera, Alvin $M . i$ Interaction of health-care professionals with critically ill children and their parents Clinical Pediatrics $1982 ; 21: 540-544$

17. Vielo, F.; Korsch, B.: Morris, M.: Gaps in doctorpatient communication. New Engl J Med 1969; 280: 335-540.

18. Spitz. A,: fil primer año de vida del riño $1 \mathrm{II}$. Edición Madrid Editorial Aguilar, 1978. 